\title{
From Synapse to Nucleus and Back Again-Communication over Distance within Neurons
}

\author{
Mike Fainzilber, ${ }^{1}$ Vivian Budnik, ${ }^{2}$ Rosalind A. Segal, ${ }^{3,4}$ and Michael R. Kreutz ${ }^{5}$ \\ ${ }^{1}$ Department of Biological Chemistry, Weizmann Institute of Science, Rehovot 76100, Israel, ${ }^{2}$ Department of Neurobiology, University of Massachusetts Medical \\ School, Worcester, Massachusetts 01605, ${ }^{3}$ Department of Pediatric Oncology and Cancer Biology, Dana-Farber Cancer Institute, and ${ }^{4}$ Department of Neurobiology, \\ Harvard Medical School, Boston, Massachusetts 02215, and 5 Neuroplasticity Group, Leibniz Institute for Neurobiology, Magdeburg 39118, Germany
}

How do neurons integrate intracellular communication from synapse to nucleus and back? Here we briefly summarize aspects of this topic covered by a symposium at Neuroscience 2011. A rich repertoire of signaling mechanisms link both dendritic terminals and axon tips with neuronal soma and nucleus, using motor-dependent transport machineries to traverse the long intracellular distances along neuronal processes. Activation mechanisms at terminals include localized translation of dendritic or axonal RNA, proteolytic cleavage of receptors or second messengers, and differential phosphorylation of signaling moieties. Signaling complexes may be transported in endosomes, or as nonendosomal complexes associated with importins and dynein. Anterograde transport of RNA granules from the soma to neuronal processes, coupled with retrograde transport of proteins translated locally at terminals or within processes, may fuel ongoing bidirectional communication between soma and synapse to modulate synaptic plasticity as well as neuronal growth and survival decisions.

\section{Introduction}

Neurons extend extremely long processes that pose a unique challenge for intracellular signaling in both vertebrates and invertebrates, requiring efficient transport mechanisms to move macromolecules and metabolites from the cell body to neurite terminals and back (Kam et al., 2009; Twiss and Fainzilber, 2009). The intracellular distances that separate soma from terminals raise important but largely unanswered questions such as, How are synaptic events translated into changes in gene expression at the distant nucleus? How can neuronal somata respond to survival or growth signals that are elicited at remote processes? Conversely, how are terminals and synapses differentially affected by transcription or translation events in the soma? Well characterized events, including membrane depolarization and calcium influx, contribute to information transfer along neuronal processes via electrophysiological encoding (Cohen and Greenberg, 2008), while physical (but slower) transfer of macromolecules has long been thought to serve primarily metabolic and housekeeping roles. However, recent studies in diverse systems have started to challenge this distinction, by revealing how localized translational and posttranslational mechanisms can combine with intracellular transport mechanisms to integrate communication among synapse,

\footnotetext{
Received Aug. 4, 2011; revised Sept. 14, 2011; accepted Sept. 15, 2011.

We gratefully acknowledge grant support as follows: M.F.--from the Israel Science Foundation, the GermanIsraeli Foundation, the Minerva Foundation, the International Foundation for Research in Paraplegia, the Christopher and Dana Reeve Foundation, and the Dr. Miriam and Sheldon G. Adelson Medical Research Foundation; V.B.—NIH Grant R01 NS063228; R.A.S.—NIH Grants R01 MH091662 and R01 NS050674; M.R.K.Center for Brain and Behavior Sciences Magdeburg, Deutsche Forschungsgemeinschaft, Bundesministerium für Bildung und Forschung, DZNE Magdeburg, the Deutsche-Israelische Projektkooperation, Leibniz Foundation (Pakt für Forschung), and Schram Foundation.

Correspondence should be addressed to Mike Fainzilber at the above address. E-mail: mike. fainzilber@weizmann.ac.il.

DOI:10.1523/JNEUROSCI.4006-11.2011

Copyright $\odot 2011$ the authors $\quad 0270-6474 / 11 / 3116045-04 \$ 15.00 / 0$
}

processes, and soma (Jordan and Kreutz, 2009; Perry and Fainzilber, 2009). This minireview briefly describes examples highlighted by the symposium, demonstrating that these mechanisms are fundamental for diverse neuronal functions.

\section{Synaptonuclear communication via posttranslational modifications}

Synapses communicate with the nucleus via multiple signaling pathways (Fig. 1), and it is generally believed that synaptic activity regulates gene expression required for long-term structural changes in synaptodendritic input (Cohen and Greenberg, 2008). In recent years, a number of potential synaptonuclear protein messengers such as Abi-1, AIDA-1D, CREB2, Jacob, Nf $\kappa \mathrm{B}$, and the Wnt receptors Frizzled and Ryk/Derailed have been identified (Jordan and Kreutz, 2009; Budnik and Salinas, 2011). Some of these messengers harbor a nuclear localization signal and require binding of neuronal importins for long-distance trafficking and nuclear import (Mosca and Schwarz, 2010). Neuronal importins are present in axons, dendrites, and synapses (Hanz et al., 2003; Thompson et al., 2004) and appear to play a crucial role as adaptor proteins for macromolecular protein complexes that are transported along microtubules. NMDA receptors (NMDARs) trigger signaling cascades in several of the above pathways and make an essential contribution to activity-dependent gene expression (Jordan and Kreutz, 2009). Intriguingly, NMDARs are present at both synaptic and extrasynaptic sites, and the subcellular localization of each receptor profoundly affects the nuclear response to its activation (Hardingham and Bading, 2010). Activation of synaptic NMDARs induces the expression of cell survival and plasticity genes, while in contrast, activation of extrasynaptic NMDARs primarily drives expression of cell death genes, linking the pathway to neurodegenerative disorders (Hardingham and Bading, 2010). An outstanding issue is, how 


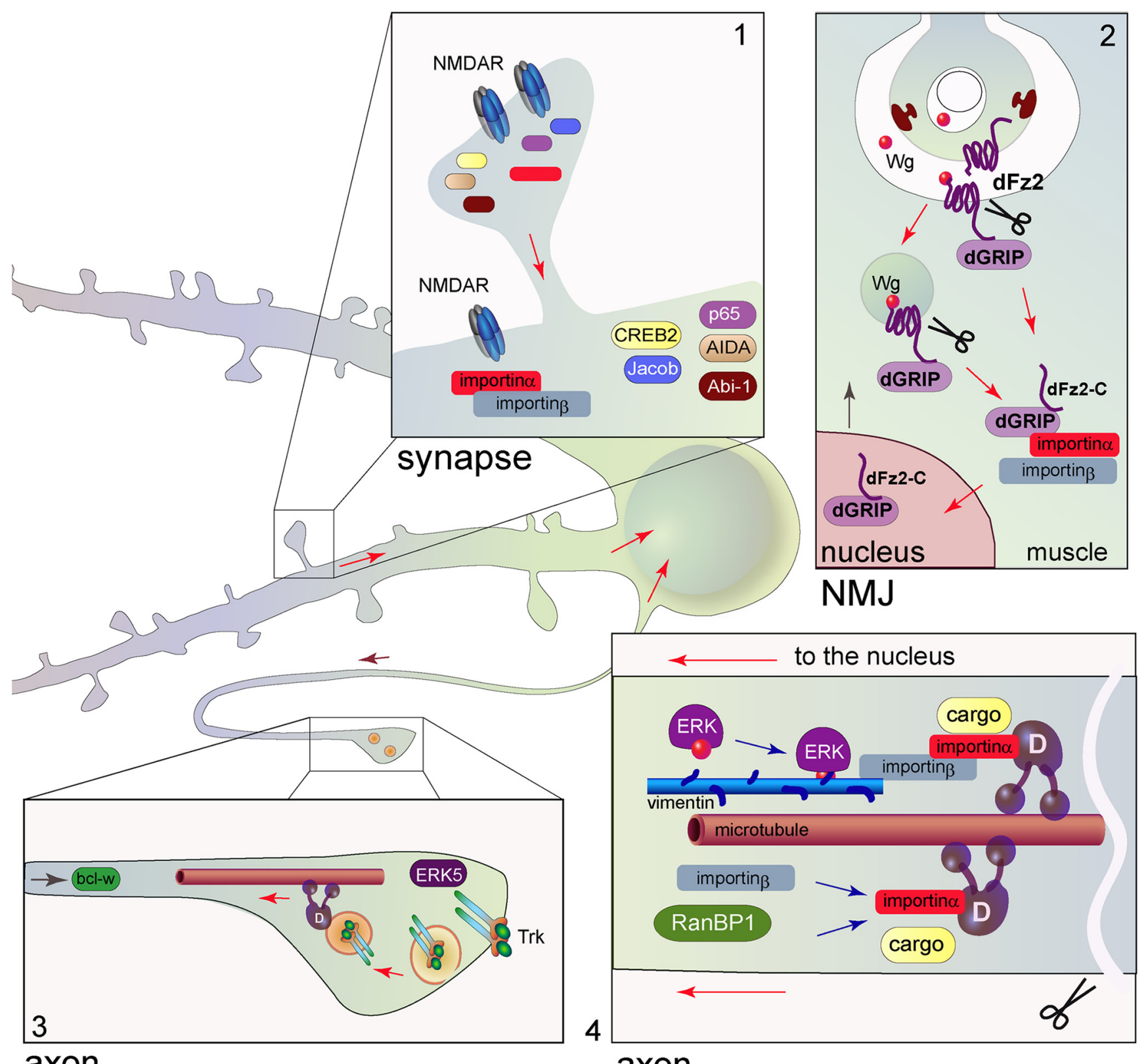

axon

axon

Figure 1. Schematic depiction of the different routes of long-distance signaling in neurons. Non-endosomal transport of macromolecular complexes frequently requires binding of importin $\alpha$ to the cargo as in synapse-to-nucleus (1), neuromuscular junction (NMJ)-to-nucleus (2), or axon-to-nucleus (4) communication. 1, A number of synaptonuclear protein messengers have been identified in recent years that can translocate to the nucleus in an activity-dependent manner. NMDA receptor activation plays a pivotal role for this type of communication. 2, Studies at the NMJ suggest that long-distance Wnt signaling is involved in the expression of genes that are required for building the postsynaptic apparatus. 3, The most investigated example of neuronal long-distance communication is probably neurotrophin signaling via Trk-containing endosomes in axons. This type of signaling involves MEF2D-regulated gene expression, including regulating expression of $\mathrm{BCl}-\mathrm{w}, \mathrm{a} \mathrm{BCl}-2$ family member that is crucial for axonal integrity in sensory neurons. 4, Following axon injury, retrograde transport of certain injury signaling molecules is based on direct interactions of cargo proteins with importin nuclear transport factors in complex with the retrograde motor dynein. In addition to importin $\beta 1$, other components or regulators of this complex are also locally translated in axons after injury, such as vimentin, a binding adaptor for phosphorylated ERK, and RanBP1, a regulator of the importins-Ran system.

can the distant nucleus discriminate between synaptic and extrasynaptic NMDAR-induced signals?

Examination of a number of proteins downstream of NMDAR has directed attention to Jacob, a recently identified Caldendrin-binding protein that translocates to neuronal nuclei following activation of NMDAR and calpain-dependent cleavage of its myristoylated $\mathrm{N}$ terminus (Dieterich et al., 2008; Kindler et al., 2009). Extrasynaptic NMDAR activation drives Jacob efficiently to the nucleus, inducing sustained dephosphorylation and transcriptional inactivation of CREB (Dieterich et al., 2008). Ja- cob also translocates to the nucleus in CA1 neurons after Schaffer collateral-dependent long-term potentiation (LTP), and hence it acts as a messenger for both synaptic and extrasynaptic NMDAR pathways (Behnisch et al., 2011). In very recent research that will be presented at the symposium, differential phosphorylation of Jacob is shown to be a key discriminating factor for these two pathways. ERK-dependent phosphorylation of a critical residue in Jacob encodes synaptic versus extrasynaptic localization of the originating NMDAR, allowing distinction between the two pathways in the nucleus. 
Another effect of NMDAR activation is to increase expression of secreted ligands from the Wnt family (Wayman et al., 2006), which have important roles in formation and plasticity of synapses (Budnik and Salinas, 2011). Conversely, secreted Wnts may have acute effects on synaptic NMDAR currents, as well as adjusting the threshold for synaptic potentiation (Cerpa et al., 2011). The molecular mechanisms regulating Wnt circuits via synaptonuclear communication are becoming increasingly clear through a series of studies on the developing neuromuscular junction (Korkut and Budnik, 2009). For example, at the Drosophila larval neuromuscular junction, the postsynaptic Frizzled Nuclear Import Wnt signaling pathway is required for the formation of the postsynaptic apparatus and for the recruitment of postsynaptic proteins as new synaptic boutons are formed during development (Ataman et al., 2008). In this pathway, the Wnt receptor Frizzled-2 (DFz2) is endocytosed by the postsynaptic muscle cell and a $\mathrm{C}$ terminal cleavage fragment is then translocated to the nucleus (Mathew et al., 2005). An analogous pathway has been described during the differentiation of cortical neurons by the atypical Wnt receptor Ryk/Derailed (Lyu et al., 2008). Alterations in the Frizzled Nuclear import pathway interfere with the proliferation of synaptic boutons during neuromuscular junction expansion, the formation of active zones, and the recruitment of postsynaptic proteins. The PDZ protein dGRIP associates with DFz2 in a vesicular pathway allowing its trafficking to the nucleus, thereby providing a direct link from the postsynaptic membrane to the postsynaptic nucleus during synapse formation (Ataman et al., 2006). As discussed in the symposium, the nuclear localization of DFz2 plays an important role in regulating transcripts required for building the postsynaptic apparatus and uncovers a novel mechanism by which synaptic signals are conveyed to the nucleus, which signals back to synapses promoting their growth and differentiation.

Together, the above findings show how posttranslational modification (via proteolysis and/or phosphorylation) of proteins resident in synapse or dendrite can facilitate their nuclear translocation, conveying both spatial and activity state information from synapse to nucleus. In the anterograde direction, the nucleus in turn exerts profound effects on synapse composition and function, most obviously by changing the expression of synaptic proteins. This type of communication is further highlighted by the targeted export of specific mRNAs from the soma to dendrites, and their regulated local translation (Donnelly et al., 2010). A plethora of different mRNAs have been found in dendrites (Swanger and Bassell, 2011), including Jacob-encoding mRNAs in the hippocampus (Kindler et al., 2009). A cis-acting dendritic-targeting element is found in the $3^{\prime}$-untranslated region (UTR) of Jacob mRNAs, and Jacob transcripts are associated with both an anterograde kinesin motor and the RNA-targeting fragile X mental retardation protein. These data suggest that local synthesis can replenish dendritic Jacob pools after NMDARinduced translocation of Jacob to the nucleus (Kindler et al., 2009).

\section{Axonuclear communication-from terminal to nucleus}

Axon-nucleus communication must overcome distances that are orders of magnitude longer than those in dendrites. Yet, paradoxically, neurotrophin signaling along axons is one of the best understood long-distance communication mechanisms in the nervous system (Cosker et al., 2008). In the most widely accepted model for this type of signaling, neurotrophins released from target tissues bind and activate Trk receptors at nerve terminals. The ligand-receptor complex is then internalized, forming a signaling endosome that traffics retrogradely from distal axons to neuronal cell bodies by motor-dependent transport along microtubules. The endosomes are transported in association with downstream signaling components such as Erk5 (Watson et al., 2001), which help to encode the spatial location of the initiating signal (Cosker et al., 2008). Arrival of the signaling complex to the cell body activates downstream transcriptional responses that define the phenotypic outcome of the signal. Recent work has examined the nature of specific transcriptional responses to retrogradely transported neurotrophins in sensory neurons, by comparing cell body versus distal axon application of the ligand (Pazyra-Murphy et al., 2009). A set of retrograde response genes was identified, including the transcription factor MEF2D, which is specifically induced by distal axon stimulation. MEF2D regulates expression of Bcl-w, an anti-apoptotic Bcl-2 family member, in response to activation of Trk-dependent ERK5/MEF2 signaling (Pazyra-Murphy et al., 2009). This pathway promotes sensory neuron survival, and moreover, Bcl-w is subsequently found to be critical for ongoing maintenance of nociceptive sensory axons (Courchesne et al., 2011). Bcl-w(-/-) mice demonstrate an adult-onset progressive nociceptor denervation without cell body loss, indicating that lack of Bcl-w results in a primary axonopathy. Furthermore, Bcl-w is enriched in axons of sensory neurons and is required to prevent axonal loss (Courchesne et al., 2011). Together, these data reveal a bidirectional mechanism wherein retrograde neurotrophin signaling from the axon activates a specific transcriptional program that in turn induces expression of an axon-targeted protein critical for axonal maintenance. The mechanisms underlying localized upregulation of Bcl-w in the axon are of keen current interest, and recent findings in this area will be presented at the symposium.

Another long-distance signaling mechanism with potentially critical roles in adult neurons is retrograde injury signaling from axonal lesion sites. Regeneration of lesioned peripheral nerves is dependent on transcriptional events in the neuronal cell body following an injury event in the axon. Studies in peripheral sensory neurons have shown that retrogradely transported injury signals are required for initiation of a regeneration response (Rishal and Fainzilber, 2010). Interestingly, retrograde transport of certain injury signaling molecules might not necessarily require endosomes (although see Abe and Cavalli, 2008 for a review covering endosomes in injury signaling), and an alternative mechanism has been proposed based on direct interactions of cargo proteins with importin nuclear transport factors complexed with molecular motors (Hanz et al., 2003). In rodent sciatic nerve, a number of importin $\alpha$ s were found in axons of both control and injured nerve in constitutive association with the retrograde motor dynein, while importin $\beta 1$ protein was present only after injury (Hanz et al., 2003). Interestingly, mRNA for importin $\beta 1$ is found in sensory axons, and is locally translated at the injury site after lesion (Hanz et al., 2003). This leads to the formation of importin $\alpha / \beta$ heterodimers bound to dynein, thereby enabling transport of signaling cargos that bind to the importin complex. Other components of the system are also locally translated in axons, specifically vimentin, which acts as a binding adaptor for phosphorylated ERK (Perlson et al., 2005), and RanBP1, which serves a regulatory role in the system (Yudin et al., 2008). However, despite proteomic identification of a plethora of signaling molecules in injured axons (Michaelevski et al., 2010), specific retrograde injury signaling cargos that bind directly to importins have not yet been described. Transcription factors (TFs) are adapted to take advantage of nucleocytoplasmic transport mechanisms; hence, they are intriguing candidates for importin-dependent retrograde cargo molecules (Perry and 
Fainzilber, 2009). A comprehensive analysis of the links between axonal injury signaling and cell body transcriptional responses in the sciatic nerve-DRG system recently implicated 39 TFs in the sensory neuron response to nerve injury (Michaelevski et al., 2010). The likelihood for direct involvement of TF cargos in retrograde injury signaling will be addressed in the symposium.

\section{Concluding remarks}

The emerging theme summarized above is that synapses, dendrites, axons, and soma are linked together by bidirectional communication mechanisms that enable information transfer encoded by macromolecules. So far, these mechanisms have been addressed primarily in the context of survival or injury signaling in axons, versus activity-based pathways in dendrites. However, recent studies describing a role for local translation in nociceptive axons (Jiménez-Díaz et al., 2008; Melemedjian et al., 2010) and in presynaptic terminals (Akins et al., 2009), raise the intriguing possibility that bidirectional transport and localized regulation might enable activity-based signaling in axons as well as dendrites. Conversely, it will be interesting to find out if the retrograde injury signaling mechanisms described in axons are activated in similar ways in lesioned dendrites. Overall, the findings presented above suggest that anterograde transport of mRNAs into processes coupled with retrograde transport of their locally translated protein products is one shared example of a mechanism allowing bidirectional information transfer between soma and terminals in neurons. The unique spatial challenges of neuronal morphology have been a driving force for the evolution of mechanisms for long-distance signaling. It will be interesting in the future to find out if the same long-distance signaling mechanisms actually provide the limiting factors for neuronal growth and size determination.

\section{References}

Abe N, Cavalli V (2008) Nerve injury signaling. Curr Opin Neurobiol $18: 276-283$.

Akins MR, Berk-Rauch HE, Fallon JR (2009) Presynaptic translation: stepping out of the postsynaptic shadow. Front Neural Circuits 3:17.

Ataman B, Ashley J, Gorczyca D, Gorczyca M, Mathew D, Wichmann C, Sigrist SJ, Budnik V (2006) Nuclear trafficking of Drosophila Frizzled-2 during synapse development requires the PDZ protein dGRIP. Proc Natl Acad Sci U S A 103:7841-7846.

Ataman B, Ashley J, Gorczyca M, Ramachandran P, Fouquet W, Sigrist SJ, Budnik V (2008) Rapid activity-dependent modifications in synaptic structure and function require bidirectional Wnt signaling. Neuron 57:705-718.

Behnisch T, Yuanxiang P, Bethge P, Parvez S, Chen Y, Yu J, Karpova A, Frey JU, Mikhaylova M, Kreutz MR (2011) Nuclear translocation of Jacob in hippocampal neurons after stimuli inducing long-term potentiation but not long-term depression. PLoS One 6:e17276.

Budnik V, Salinas PC (2011) Wnt signaling during synaptic development and plasticity. Curr Opin Neurobiol 21:151-159.

Cerpa W, Gambrill A, Inestrosa NC, Barria A (2011) Regulation of NMDAreceptor synaptic transmission by Wnt signaling. J Neurosci 31:9466-9471.

Cohen S, Greenberg ME (2008) Communication between the synapse and the nucleus in neuronal development, plasticity, and disease. Annu Rev Cell Dev Biol 24:183-209.

Cosker KE, Courchesne SL, Segal RA (2008) Action in the axon: generation and transport of signaling endosomes. Curr Opin Neurobiol 18:270-275.

Courchesne SL, Karch C, Pazyra-Murphy MF, Segal RA (2011) Sensory neuropathy attributable to loss of Bcl-w. J Neurosci 31:1624-1634.

Dieterich DC, Karpova A, Mikhaylova M, Zdobnova I, König I, Landwehr M, Kreutz M, Smalla KH, Richter K, Landgraf P, Reissner C, Boeckers TM, Zuschratter W, Spilker C, Seidenbecher CI, Garner CC, Gundelfinger ED, Kreutz MR (2008) Caldendrin-Jacob: a protein liaison that couples NMDA receptor signalling to the nucleus. PLoS Biol 6:e34.
Donnelly CJ, Fainzilber M, Twiss JL (2010) Subcellular communication through RNA transport and localized protein synthesis. Traffic 11:1498-1505.

Hanz S, Perlson E, Willis D, Zheng JQ, Massarwa R, Huerta JJ, Koltzenburg M, Kohler M, van-Minnen J, Twiss JL, Fainzilber M (2003) Axoplasmic importins enable retrograde injury signaling in lesioned nerve. Neuron 40:1095-1104.

Hardingham GE, Bading H (2010) Synaptic versus extrasynaptic NMDA receptor signalling: implications for neurodegenerative disorders. Nat Rev Neurosci 11:682-696.

Jiménez-Díaz L, Géranton SM, Passmore GM, Leith JL, Fisher AS, Berliocchi L, Sivasubramaniam AK, Sheasby A, Lumb BM, Hunt SP (2008) Local translation in primary afferent fibers regulates nociception. PLoS One 3:e1961.

Jordan BA, Kreutz MR (2009) Nucleocytoplasmic protein shuttling: the direct route in synapse-to-nucleus signaling. Trends Neurosci 32:392-401.

Kam N, Pilpel Y, Fainzilber M (2009) Can molecular motors drive distance measurements in injured neurons? PLoS Comput Biol 5:e1000477.

Kindler S, Dieterich DC, Schütt J, Sahin J, Karpova A, Mikhaylova M, Schob C, Gundelfinger ED, Kreienkamp HJ, Kreutz MR (2009) Dendritic mRNA targeting of Jacob and N-methyl-d-aspartate-induced nuclear translocation after calpain-mediated proteolysis. J Biol Chem 284:25431-25440.

Korkut C, Budnik V (2009) WNTs tune up the neuromuscular junction. Nat Rev Neurosci 10:627-634.

Lyu J, Yamamoto V, Lu W (2008) Cleavage of the Wnt receptor Ryk regulates neuronal differentiation during cortical neurogenesis. Dev Cell 15:773-780.

Mathew D, Ataman B, Chen J, Zhang Y, Cumberledge S, Budnik V (2005) Wingless signaling at synapses is through cleavage and nuclear import of receptor DFrizzled2. Science 310:1344-1347.

Melemedjian OK, Asiedu MN, Tillu DV, Peebles KA, Yan J, Ertz N, Dussor GO, Price TJ (2010) IL-6- and NGF-induced rapid control of protein synthesis and nociceptive plasticity via convergent signaling to the eIF4F complex. J Neurosci 30:15113-15123.

Michaelevski I, Segal-Ruder Y, Rozenbaum M, Medzihradszky KF, Shalem O, Coppola G, Horn-Saban S, Ben-Yaakov K, Dagan SY, Rishal I, Geschwind DH, Pilpel Y, Burlingame AL, Fainzilber M (2010) Signaling to transcription networks in the neuronal retrograde injury response. Sci Signal 3:ra53.

Mosca TJ, Schwarz TL (2010) The nuclear import of Frizzled2-C by Importins-beta11 and alpha2 promotes postsynaptic development. Nat Neurosci 13:935-943.

Pazyra-Murphy MF, Hans A, Courchesne SL, Karch C, Cosker KE, Heerssen HM, Watson FL, Kim T, Greenberg ME, Segal RA (2009) A retrograde neuronal survival response: target-derived neurotrophins regulate MEF2D and bcl-w. J Neurosci 29:6700-6709.

Perlson E, Hanz S, Ben-Yaakov K, Segal-Ruder Y, Seger R, Fainzilber M (2005) Vimentin-dependent spatial translocation of an activated MAP kinase in injured nerve. Neuron 45:715-726.

Perry RB, Fainzilber M (2009) Nuclear transport factors in neuronal function. Semin Cell Dev Biol 20:600-606.

Rishal I, Fainzilber M (2010) Retrograde signaling in axonal regeneration. Exp Neurol 223:5-10.

Swanger SA, Bassell GJ (2011) Making and breaking synapses through local mRNA regulation. Curr Opin Genet Dev 21:414-421.

Thompson KR, Otis KO, Chen DY, Zhao Y, O'Dell TJ, Martin KC (2004) Synapse to nucleus signaling during long-term synaptic plasticity; a role for the classical active nuclear import pathway. Neuron 44:997-1009.

Twiss JL, Fainzilber M (2009) Ribosomes in axons-scrounging from the neighbors? Trends Cell Biol 19:236-243.

Watson FL, Heerssen HM, Bhattacharyya A, Klesse L, Lin MZ, Segal RA (2001) Neurotrophins use the Erk5 pathway to mediate a retrograde survival response. Nat Neurosci 4:981-988.

Wayman GA, Impey S, Marks D, Saneyoshi T, Grant WF, Derkach V, Soderling TR (2006) Activity-dependent dendritic arborization mediated by CaM-kinase I activation and enhanced CREB-dependent transcription of Wnt-2. Neuron 50:897-909.

Yudin D, Hanz S, Yoo S, Iavnilovitch E, Willis D, Gradus T, Vuppalanchi D, Segal-Ruder Y, Ben-Yaakov K, Hieda M, Yoneda Y, Twiss JL, Fainzilber M (2008) Localized regulation of axonal RanGTPase controls retrograde injury signaling in peripheral nerve. Neuron 59:241-252. 